Service social

\title{
Les tendances actuelles des services aux enfants et à leur famille
}

\section{André Beaudoin}

Volume 47, numéro 3-4, 1998

Enfance : enjeux et réalités 2000

URI : https://id.erudit.org/iderudit/706793ar

DOI : https://doi.org/10.7202/706793ar

Aller au sommaire du numéro

Éditeur(s)

École de service social de l'Université Laval

ISSN

1708-1734 (numérique)

Découvrir la revue

Citer ce document

Beaudoin, A. (1998). Les tendances actuelles des services aux enfants et à leur famille. Service social, 47(3-4), 11-13. https://doi.org/10.7202/706793ar d'utilisation que vous pouvez consulter en ligne.

https://apropos.erudit.org/fr/usagers/politique-dutilisation/ 


\section{AVANT-PROPOS}

\section{Les tendances actuelles des services aux enfants et à leur famille}

André BEAUDOIN

La place faite dans la société à la santé, à l'éducation et au bienêtre de l'enfance et de la jeunesse est garante de leur avenir. La Déclaration mondiale de 1990 en faveur de la survie, de la protection et du développement des enfants met bien en évidence cette réalité. II faut faire tous les efforts nécessaires pour favoriser le développement des enfants sur les plans physique, psychologique et social, assurer leur éducation, prévenir les problèmes et trouver les solutions requises aux différentes difficultés qu'ils peuvent éprouver. La famille, l'environnement et leurs différents systèmes d'appui, qu'il s'agisse du système scolaire, du système de garantie des droits, des soins de santé ou des services, exercent une influence déterminante.

Tout en se situant dans cette perspective d'ensemble, ce numéro de Service social qui porte surtout sur l'enfance se situe dans le contexte des enjeux et des réalités auxquels doivent faire face les services sociaux devant les difficultés vécues par plusieurs jeunes, qu'il s'agisse d'enfants ou d'adolescents, dans leur famille et dans leurs autres systèmes d'appartenance.

Dans le premier article, Oscar d'Amours, tenant compte de l'évolution des droits de l'enfance et de la jeunesse, analyse dans son ensemble l'évolution du modèle de protection, qui met l'accent sur la réhabilitation et sur les besoins des jeunes, surtout des délinquants, et celle du modèle de justice, qui accorde une plus grande importance au processus légal en insistant sur la responsabilisation du jeune. Son analyse de l'évolution et de la réalité actuelle le conduit à affirmer qu'il est nécessaire de tenir

Service social, vol. 47, nos 3 et 4, 1998-1999 
compte davantage des victimes dans une perspective de partenariat entre les différents intervenants dans un contexte de participation communautaire. Selon Dagenais, Bouchard et Turner, qui rapportent les résultats d'une recherche-action à laquelle ont participé des intervenants, ces derniers doivent travailler dans un contexte caractérisé par le stress, la violence et la gravité des situations qui sont leur quotidien en matière de protection. La plupart du temps ils ne peuvent pas compter sur un soutien adéquat pour y faire face et la concertation avec des acteurs multiples paraît souvent problématique.

Les quatre articles suivants examinent autant d'aspects internes du processus d'intervention. Saint-Jacques et ses collaborateurs se penchent sur les pratiques d'implication parentale en centre jeunesse et mettent en évidence le rôle crucial joué par les intervenants. Poirier et ses collaborateurs s'intéressent aux principaux facteurs associés à la fréquence et à la durée des contacts entre les enfants de moins de 14 ans placés en famille d'accueil et leurs parents. Mireault et ses collaborateurs font par ailleurs l'évaluation d'un programme visant à impliquer les parents pendant le placement des adolescents en centre de réadaptation. En somme, trois aspects de l'implication parentale en lien avec l'intervention sont l'objet d'attention: le rôle des parents en cours d'intervention, la présence et le maintien des contacts parents-enfants pendant le placement en famille d'accueil et l'insertion des parents dans la réadaptation en centre d'accueil. Dans le quatrième article, Turcotte et ses collaboratrices tracent un portrait des interventions auprès des enfants et adolescents exposés à la violence conjugale. Tout en montrant que ces interventions identifient l'approche féministe comme assise théorique, le choix des méthodes d'intervention serait davantage lié aux conditions de pratique dans lesquelles se trouvent les intervenantes, ce qui rend difficile l'utilisation de l'intervention de groupe, par exemple.

L'article de Claude Larivière, à la lumière des résultats de la première étape d'une étude portant sur l'entente cadre conclue entre les centres jeunesse et les CLSC du Québec, examine le continuum d'accessibilité aux services à l'enfance qui en découle.

Dans le premier des deux autres articles sur cette thématique, Bouchard et ses collaborateurs font ressortir que la dynamique familiale peut vraiment avoir une influence sur la réussite scolaire, 
si elle s'accompagne d'un certain nombre de conditions socioéconomiques de base. Marc Boily et ses collaboratrices font enfin ressortir comment les problèmes de santé mentale des parents qui ne bénéficient pas d'un traitement approprié constituent des facteurs de négligence parentale et de mauvais traitements et sont à l'origine d'autres problèmes qui ont une influence importante dans l'exercice des rôles parentaux.

La contribution internationale du présent numéro, fournie par Maïmouna Sourang, André Beaudoin et Élaine Carey-Bélanger, porte sur l'implication économique et l'intégration sociale des femmes bénéficiaires d'une caisse d'épargne et de crédit au Sénégal.

Sous la rubrique Recherches et documents, enfin, Jean-Pierre Deslauriers et Richard Sévigny nous rappellent le rôle de l'organisation communautaire au temps de l'exclusion et de l'économie sociale. 
